\title{
Plant-Parasitic Nematodes on Stone FRUITS AND CITRUS IN LEBANON
}

Said K. Ibrahim, Ibrahim Azar, Christian Naser, Badran Akikki and Ludmilla Ibrahim Faculty of agricultural sciences, Lebanese University, Beirut, Lebanon

sibarhim58@yahoo.com

(Received 14 April 2015 - Accepted 11 August 2015)

\section{ABSTRACT}

Ibrahim Said K., Ibrahim Azar, Christian Naser, Badran Akikki and Ludmilla Ibrahim. 2016. Plant-parasitic nematodes on stone fruits and citrus in Lebanon. Lebanese Science Journal, 17(1): 9-24.

This study aimed to determine the occurrence, distribution of plant parasitic nematodes on stone fruits in Lebanon and to determine the effect of plant extracts on the mortality of several nematode species. A total of 308 soil samples were collected from five different crops. Almost all surveyed areas showed infection with nematodes. The soil infestation rate with nematodes in collected soil samples from all 10 surveyed crops ranged from 66.6 to $100 \%$. Eighteen out of 308 soil samples were free of nematodes (5.8\%). All the collected soil samples from nectarine and plum orchards were infested with nematodes (100\%), followed by citrus (97.6\%), apple (88.7\%), pear and quince (85.7\%), and cherry $(81.4 \%)$. The lowest infection (66.6\%) was detected on almond and apricot. The level of infestation varied from one area to another and ranged between 0.1 and 28 nematodes per 1 $g$ of soil, with the highest number obtained on cherry. Several genera were identified based on morphological characters including: root-knot nematodes (Meloidogyne spp.), Tylenchulus, Xiphinema, Rotylenchus, Pratylenchus, and Longidorus. Tylenchulus and Radopholus spp. were the most common on citrus trees, whereas Pratylechus and Meloidogyne spp. were detected almost in all the samples collected from all the crops. Six chopped aromatic plants were tested in pot experiments to control nematodes population densities. The results revealed that carbofuran (nematicide) was the most effective (88.48\%) in comparison to the plant materials. Allium sativum gave the highest control $(76.52 \%)$ followed by Tageta patula (72.0\%), Cucurbita maxima (71.84\%) and Inula viscosa (63.96\%). Origanum syriacum (55.04\%) and Thymus (53.72\%) were less effective in comparison to the rest of tested plant materials.

Keywords: Cucurbita maxima, Inula viscosa, Origanum syriacum, plant extracts, plant parasitic nematodes, soil survey, stone fruits, Tageta patula, Thymus capitatus

\section{INTRODUCTION}

The Lebanese agricultural sector is suffering from several serious problems. Pests and diseases are one of those causing annual losses of millions of dollars to the economy every year. They attack a wide range of economically important crops of horticultural, agricultural and forest systems. Food security concern has always been a priority. Citrus,

http://dx.doi.org/10.22453/LSJ-017.1.009024

National Council for Scientific Research - Lebanon 2016@ 
apple and cherry play an important role in the county's economy in terms of export and local demand.

Today plant parasitic nematodes are recognized as major agricultural pathogens and are known to attack plants and cause crop losses throughout the world. Some estimates suggest they cause 77 billion dollars of damage worldwide each year (Sasser \& Freckman, 1987). Several species of plant parasitic nematode are considered as a serious problem in citrus and apple production worldwide (Nyczepir \& Becker, 1998). They damage the plant by directly attacking roots and subsequently predisposing them to secondary infections by bacteria and fungi and other pathogens. The most economically important species belong to the genera Criconemella, Meloidogyne, Pratylenchus, Longidorus, Radopholus, Xiphinema, Trichodorus and Paratrichodorus, and are widely distributed in fruit orchards throughout the world (Nyczepir \& Halbrendt, 1993; Nyczepir \& Becker, 1998). Forty plant parasitic nematode species were identified in soil of fruit orchards in the southeastern and southwestern areas of Slovakia (Lišková et al., 2007). Nine different species of Pratylenchus were associated with stone fruits (Duncan, 2005). Meloidogyne hapla was detected on avocado roots in New Zealand (Knight, 2001) and Helicotylenchus dihystera reduced seedling growth by $20-50 \%$ (Saltaren et al., 1999). In most cases the damage caused by nematode is, however, not easily visible. For example, peaches are one of the few crops that can die from nematode's damage. It is particularly younger trees less than 18 months old that can die due to root-knot nematodes. Parasitic nematodes (ring, lesion, root-knot nematodes, and dagger nematodes on some rootstocks) cause root damage in some of California's almond and stone fruit orchards, and the ring nematode has been associated with the bacterial canker complex on sandy soils. Prunus replant disease (PRD) occurs widely in California, causing growth suppression and, in severe cases, tree death (Tudor \& McKenry, 1989).

The possibilities for controlling nematodes on stone fruits are limited because in most systems of cultivation they are grown as a permanent crop and there are no nematode resistant varieties. Also, there is no specific nematode control methods are yet practiced in traditional cultivations where crop debris including grass, coffee, oil palm and cocoa husk are used in some countries. The effect of mulches on nematode populations is not clear and the critical evaluation of the use of organic materials in orchards plantations is long overdue.

Management of plant parasitic nematodes could be very difficult and costly especially in not-yet-established orchards. Certain plants are able to kill or repel pests, disrupt their lifecycle, or discourage them from feeding. The most widely used approach, for the management of nematodes, is the use of chemical control. However, many of these chemicals have been discontinued or suspended its use by the Environmental Protection Agency or the manufacturing companies. There is need to evaluate the use of environmentally-sound methods for nematodes control as components of an integrated pest management (IPM) system. Plant types particularly suitable for biofumigation including the family Brassicaceae (cabbage, cauliflower, broccoli, kale, canola and mustard), the family Moringaceae (horseradish and certain types of radishes), and also Marigold species could be used as alternative control methods against nematodes. Aqueous extracts from California poppy (Eschscholzia papaver Chamb.), selected marigold species (Tagetes spp.), and cahaba white vetch demonstrated nematicidal activity against the ring nematode, Criconemella xenoplax in California (Tudor \& McKenry, 1989). The feasibility of applying these extracts to orchard soils is being investigated. In South Carolina, two perennial ground cover species, nimblewill 
grass (Muhlenbergia schreberi J.F. Gmel) and buckhorn plantain (Plantago lanceolata L. ) suppressed C. xenoplax when interplanted in the tree rows (Zehr et al., 1986).

Several species of nematodes are also considered the major limiting factors of stone fruits production in Lebanon. Plant extracts which contain volatile compounds, especially essential oils, have been found to possess antimicrobial, insecticidal and nematicidal activity (Thackray et al., 1990; Okoko et al., 1999) and against nematodes (Marban-Mendoza et al., 1987; Ibrahim et al., 2006; Ibrahim \& Traboulsi, 2009; Ibrahim et al., 2011; Ibrahim et al., 2012).

The current work is the first to be carried out in Lebanon in order to establish Data Bank on the occurrence and distribution of plant parasitic nematodes on stone fruits and citrus and also to determine the effect of chopped plant materials on nematodes mortality in pot experiments.

\section{MATERIALS AND METHODS}

\section{Sampling methods}

Soil samples were collected between April and July 2013-2014 from different regions of Lebanon (Fig. 1, Tables 1-8). Approximately, each orchard was divided into 1 Dunum depending on the size of the orchard. Collection of samples was carried out according to the method used by Speijer \& De Waele (1997) with some modifications. Several holes of $20 \times 20 \times 20 \mathrm{~cm}^{3}$ were dug next to the corm of the mother plant and soils were collected and placed in a marked plastic bag. Two different samples were taken from each orchard. Each sample contained about $1.5-2 \mathrm{~kg}$ of soil taken from around several plants. The top $5 \mathrm{~cm}$ soil was discarded. The samples were stored at $4^{\circ} \mathrm{C}$ until used. Nematodes were identified using morphological characters (CAB International, UK) on the genera level only.

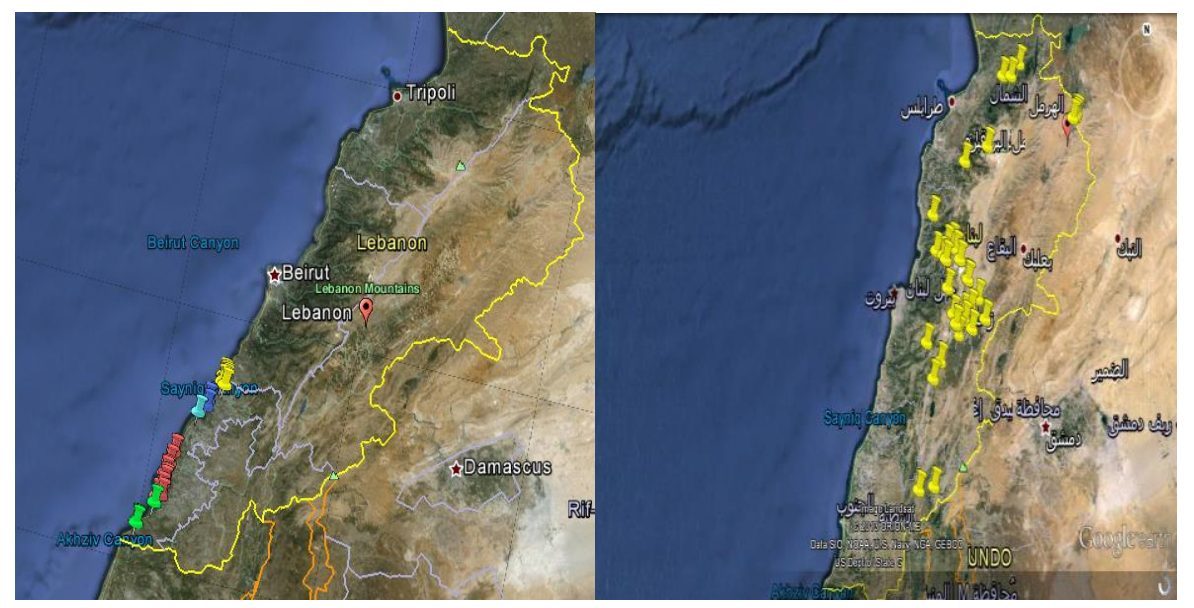

Figure 1. Maps of Lebanon showing the sampling areas on citrus in south Lebanon (left) and on apple (right). 


\section{Extraction and counting of nematodes}

The soil extraction was done as reported by Fenwick (1949). Two hundred gramms of soil were placed in the funnel and water was added slowly to moist the soil and left for 24 hours for extraction. After 24 hours, the sieve containing soil was removed, water was collected into a measuring beaker and the volume of water was recorded. Each sample was mixed well and $2 \mathrm{ml}$ suspension was transferred into a counting chamber. The number of nematodes was counted and recorded. Individual nematodes were picked up from extraction and transferred on a temporary mounts in water on a glass slide for diagnosis. Gathered nematodes were preserved in 5\% formaldehyde solution and permanently mounted with glycerine. The presence of nematodes in each sample was identified based on taxonomic characters and morphological measurements under light microscope (Hooper \& Ibrahim, 1994; Ibrahim \& Hooper, 1994). The presence of stylet in plant parasitic nematodes allows the exclusion of saprophytic or free living nematodes form plant parasitic nematodes.

\section{Impact of chopped plant materials against nematodes using pot experiment}

Infested soils with nematodes were collected from an infested orchard previously tested for nematodes infestation (Ibrahim et al., 2012). Nematodes were identified using morphological characters (CAB International) to the genera level only. Collected soils were thoroughly mixed and divided into several batches. Different plant materials including Cucurbita maxima, Inula viscosa, Origanum syriacum, Tageta patula and Thymus capitatus (Table 9) were air dried at room temperature, chopped into small pieces and $30 \mathrm{~g}$ of each treatment were mixed thoroughly with approximately $1.5 \mathrm{~kg}$ of infested soil (per replication), and then placed in 2 litre plastic pot. A nematicides (carbofuran) was used as comparative control. Each pot was planted with a small plant. Each treatment was replicated three times. The control was replicated three times and grown under the same conditions but without treatment. Pots were arranged on a bench in a randomised complete block design and placed in the open air under natural conditions. Pots were watered as needed. At 20 days interval from planting date three plants were assessed. Each plant was carefully removed from the soil and the root system was recorded for nematodes infection. Soil of each pot was thoroughly mixed and sub-samples were taken for nematodes extraction using the method described above (Fenwick, 1949).

\section{Statistical analysis}

Sigma Stat 2.0 program was used for statistical analysis and calculation of statistical parameters (mean, one way ANOVA, Tukey). Data was transformed into probate unit.

\section{RESULTS}

\section{Soil survey in cherry orchards}

In the survey of cherry trees in different parts of Lebanon, 99 soil samples were collected. Almost all of the collected soil samples (81.4\%) were infected with nematodes. The level of infestation of soil sample ranged between 0.1 and 15.9 nematodes per g soil (Table 1). Soil samples collected from Maten, Kesrwan, west Beqaa, north Beqaa, south Lebanon, Zahle and Chouf areas were all infested with nematodes (100\%). The level of infestation with nematodes population varied from one area to another. The highest level was observed ( 0.1 - 
15.9) in Kfarselwan, Chouf. While the lowest infestation (0.0-0.1) was detected in soil samples collected from Bcharre. No nematodes infection was observed in several areas such as: Taalabeya, Central Beqaa, Fnaydek, Akkar, and Bcharre area. Microscopic identification revealed several genera present in the soil samples including: root-knot nematodes (Meloidogyne spp.), Tylenchulus, Xiphinema, and Rotylenchus and free living nematodes.

TABLE 1

Numbers of Plant-Parasitic Nematodes in Soil Samples Collected from Different Locations in Cherry Orchards

\begin{tabular}{|c|c|c|c|c|c|}
\hline Region & Area & $\begin{array}{c}\text { Number } \\
\text { of } \\
\text { samples }\end{array}$ & $\begin{array}{c}\text { Range of } \\
\text { nematodes in } \\
1 \mathrm{~g} \text { of soil } \\
\end{array}$ & Mean & $\begin{array}{c}\text { Infection } \\
(\%)\end{array}$ \\
\hline \multirow{4}{*}{ Maten } & Wede El Karem & 3 & $0.1-2.0$ & 0.6 & \multirow{4}{*}{100} \\
\hline & Ain El Qabou & 1 & $0.2-0.2$ & 0.2 & \\
\hline & Sannine & 1 & $2.0-2.0$ & 2.2 & \\
\hline & Bekish & 1 & $0.8-1.5$ & 1.1 & \\
\hline \multirow[t]{5}{*}{ Kesrwan } & Qlayaat & 1 & $0.3-0.7$ & 0.5 & \multirow{5}{*}{100} \\
\hline & Blat Kfardebyen & 1 & $0.8-1.4$ & 0.9 & \\
\hline & $\begin{array}{l}\text { Choum } \\
\text { Kfardebyen }\end{array}$ & 1 & $1-1.35$ & 1.2 & \\
\hline & $\begin{array}{l}\text { Mrah Cheded } \\
\text { Kfardebyen }\end{array}$ & 3 & $0.5-2.5$ & 1.8 & \\
\hline & Zaatreh & 2 & $0.7-3.8$ & 2.1 & \\
\hline \multirow[t]{3}{*}{ West Bekaa } & Saghbine & 2 & $0.7-8.0$ & 4.3 & \multirow{3}{*}{100} \\
\hline & Ain Zebde & 1 & $3.9-3.5$ & 3.7 & \\
\hline & Qab Elias & 7 & $0.6-28$ & 12.9 & \\
\hline North Bekaa & Al Qaa & 14 & $0.4-5.6$ & 2.5 & 100 \\
\hline \multirow{7}{*}{$\begin{array}{l}\text { Central } \\
\text { Bekaa }\end{array}$} & Marej & 4 & $0.6-5.2$ & 1.9 & \multirow{7}{*}{88.8} \\
\hline & Kfar zabad & 6 & $1.8-9.8$ & 4.2 & \\
\hline & Taalabaya & 1 & $0.0-0.0$ & 0.0 & \\
\hline & Taalabaya & 4 & $0.0-1.8$ & 1.3 & \\
\hline & Meksy & 1 & $1.6-3.6$ & 2.6 & \\
\hline & Jdita & 4 & $1.6-7.0$ & 3.8 & \\
\hline & Saadneyil & 2 & $0.8-12.3$ & 5.6 & \\
\hline Zahle & Zahle & 4 & $0.7-4.8$ & 2.2 & 100 \\
\hline \multirow{2}{*}{$\begin{array}{l}\text { South } \\
\text { Lebanon }\end{array}$} & Majidiyeh & 3 & $1.5-5.6$ & 2.8 & \multirow{2}{*}{100} \\
\hline & Ain Arab & 1 & $5.0-5.2$ & 5.1 & \\
\hline \multirow[t]{4}{*}{ West Bekaa } & $\begin{array}{l}\text { Qab Elias / El } \\
\text { Bayda }\end{array}$ & 4 & $1.6-8.4$ & 4.6 & \multirow{4}{*}{100} \\
\hline & $\begin{array}{l}\text { Qab Elias /EI } \\
\text { Chaabeh }\end{array}$ & 1 & $11.8-14.9$ & 13.3 & \\
\hline & $\begin{array}{l}\text { Qab Elias / EI } \\
\text { Hamra }\end{array}$ & 1 & $5.7-5.85$ & 5.6 & \\
\hline & $\begin{array}{l}\text { Qab Elias / El } \\
\text { Talleh }\end{array}$ & 1 & $8.6-10.6$ & 9.6 & \\
\hline
\end{tabular}




\begin{tabular}{|c|c|c|c|c|c|}
\hline \multirow[t]{3}{*}{ Chouf } & Kfarselwen & 4 & $2.4-15.9$ & 6.0 & \multirow{3}{*}{100} \\
\hline & Jwar El Hawz & 2 & $1.0-2.3$ & 1.6 & \\
\hline & Barouk & 5 & $0.6-3.7$ & 1.9 & \\
\hline \multirow[t]{3}{*}{ Akkar } & Hrar & 3 & $3.5-6.4$ & 3.4 & \multirow{3}{*}{87.5} \\
\hline & Fnaydek & 1 & 0 & 0 & \\
\hline & Fnaydek & 4 & $0.7-3.9$ & 1.7 & \\
\hline Tannourine & Tanourine-Harisa & 3 & $0.0-4.0$ & 2.0 & 50 \\
\hline \multirow[t]{2}{*}{ Bcharre } & Bcharre & 0 & 0 & 0 & \multirow{2}{*}{50} \\
\hline & Bcharre & 1 & $0-0.1$ & 0.1 & \\
\hline Total & & 99 & $0.0-15.9$ & 3.0 & 81.4 \\
\hline
\end{tabular}

Soil survey in citrus orchards

In the survey on citrus trees in the south-Lebanon coast 41 samples were collected. Almost all of the collected soil samples (90\%) were infected with nematodes. The level of infestation of soil samples ranged between 0.1 and 21.3 nematodes per g soil (Table 2). Soil samples collected from the southern areas were all infested with nematodes. Samples from Saida had a $100 \%$ infestation and the number of nematodes per gram of soil ranged between 0.35 and 11.6; in Zahrani 50\% infestation was observed. A 100\% infestation in Sarafand and Sour, but the number of nematodes in soil varied between them. Several genera were identified under the microscope including: root-knot nematodes (Meloidogyne spp.), Tylenchulus, Xiphinema, Pratylenchus spp. and Rotylenchus, and free living nematodes.

TABLE 2

Numbers of Plant-Parasitic Nematodes in Soil Samples Collected from Various Locations in South Lebanon in Citrus Orchards

\begin{tabular}{|c|c|c|c|c|c|}
\hline Region & Area & $\begin{array}{c}\text { Number of } \\
\text { samples }\end{array}$ & $\begin{array}{c}\text { Range of } \\
\text { nematodes in } \\
1 \mathrm{~g} \text { of soil }\end{array}$ & Mean & $\begin{array}{c}\text { Infection } \\
(\%)\end{array}$ \\
\hline \multirow[t]{4}{*}{ Saida } & $\begin{array}{l}\text { Machrouh El } \\
\text { wakef }\end{array}$ & \multirow{4}{*}{6} & 4.6-11.6 & 8.1 & \multirow{4}{*}{100} \\
\hline & Road hobeh (2) & & $0.8-3.8$ & 2.7 & \\
\hline & Saynik (2) & & $1.9-2.6$ & 3.7 & \\
\hline & Darb EI Sim & & $0.4-0.5$ & 0.4 & \\
\hline \multirow[t]{2}{*}{ Zahrani } & Brak & \multirow{2}{*}{2} & $0.0-0.0$ & 0.0 & \multirow{2}{*}{50} \\
\hline & Jubayli project & & $2.3-4.1$ & 3.4 & \\
\hline Sarafand & Dhour sarafand (3) & 3 & $0.1-4.5$ & 2.0 & \\
\hline \multirow[t]{7}{*}{ Sour } & El Berghlier & \multirow[t]{7}{*}{25} & $2.5-2.8$ & 2.7 & \multirow{7}{*}{100} \\
\hline & Chabriha (2) & & $0.3-1.0$ & 0.6 & \\
\hline & Chawakir (2) & & $1.2-2.3$ & 3.1 & \\
\hline & Bourj chemali (2) & & $0.9-3.5$ & 2.2 & \\
\hline & Rachidyeh (2) & & $1.0-3.2$ & 2.5 & \\
\hline & Batoulaii (2) & & $0.3-4.6$ & 1.8 & \\
\hline & Alayle (2) & & $1.8-4.4$ & 4.9 & \\
\hline
\end{tabular}




\begin{tabular}{|c|c|c|c|c|c|}
\hline & El-Aiin (2) & & $2.0-6.6$ & 2.8 & \\
\hline & Zahryeh (6) & & $0.1-13.1$ & 5.3 & \\
\hline & Mansoura (2) & & $1.5-5.6$ & 5.7 & \\
\hline & Hawch (2) & & $0.7-21.3$ & 8.2 & \\
\hline Nakoura & Nakoura (3) & 5 & $1.0-9.5$ & 9.1 & \multirow{2}{*}{100} \\
\hline & Elileh (2) & & $2.5-10.1$ & 6.5 & \\
\hline Total & 19 & 41 & $0.0-21.3$ & 3.8 & 97.6 \\
\hline
\end{tabular}

() Number of samples of the same area.

\section{Soil survey in apple orchards}

Over 53 soil samples were collected from apple orchards from different parts of Lebanon (Table. 3). Six out 53 soil samples $(88.7 \%)$ were not infested with nematodes. The level of infestation of soil samples ranged between 0.1 and 16.0 nematodes per $g$ soil. $71.4 \%$ of the soil samples collected from the Maten region were infested with nematodes. The highest infection was in Bakich Chwar al-Jered (0.3-0.4). No nematodes were detected in the two samples collected from Sannine area. Samples from Keserwen area had $100 \%$ infestation and number of nematodes ranged between 0.4 and 4.30 nematodes in $1 \mathrm{~g}$ soil. Similar results $(100 \%)$ were obtained in west Bekaa with significantly higher number of nematodes $(0.2-$ 16.0) per $1 \mathrm{~g}$ soil. A total infestation (100\%) was also detected in soil samples gathered from Chouf, Akkar and Bcharreh, but the average number of nematodes varied from one area to another. Several genera were identified under the microscope including: Pratylenchus spp. root-knot nematodes (Meloidogyne spp.), Xiphinema, Longidorus and Rotylenchus. Pratylenchus and Meloidogyne spp. were the most abandoned species.

TABLE 3

Numbers of Plant-Parasitic Nematodes in Soil Samples Collected from Different Locations in Apple Orchards

\begin{tabular}{|c|c|c|c|c|c|}
\hline Region & Area & $\begin{array}{c}\begin{array}{c}\text { Number } \\
\text { of } \\
\text { samples }\end{array} \\
\end{array}$ & $\begin{array}{c}\text { Range of } \\
\text { nematodes in } \\
1 \mathrm{~g} \text { of soil }\end{array}$ & Mean & $\begin{array}{c}\text { Infection } \\
(\%)\end{array}$ \\
\hline \multirow[t]{5}{*}{ Maten } & Wede EI Karem & 2 & $0.1-1.2$ & 0.6 & \multirow{5}{*}{71.4} \\
\hline & El Machrea & 1 & $0.3-0.4$ & 0.4 & \\
\hline & Sannine & 2 & $0.0-0.4$ & 0.4 & \\
\hline & Sannine & 1 & 0.0 & 0 & \\
\hline & Bakich Chwar El & 1 & $0.3-0.4$ & 0.4 & \\
\hline \multirow[t]{3}{*}{ Keserwen } & Kolaiaat & 1 & $1.0-2.0$ & 1.5 & \multirow{3}{*}{100} \\
\hline & Kfardebyen & 6 & $0.4-4.3$ & 2.5 & \\
\hline & Zaatreh & 1 & $0.7-2.1$ & 1.4 & \\
\hline \multirow[t]{3}{*}{$\begin{array}{l}\text { West } \\
\text { Bekaa }\end{array}$} & $\begin{array}{l}\text { Saghbine(ain } \\
\text { choua) }\end{array}$ & 4 & $0.2-2.5$ & 1.1 & \multirow{3}{*}{100} \\
\hline & Qaroun & 1 & $2.0-16.0$ & 9.0 & \\
\hline & $\begin{array}{l}\text { Qab Elias/ EI } \\
\text { Talleh }\end{array}$ & 1 & $1.1-1.3$ & 1.2 & \\
\hline
\end{tabular}




\begin{tabular}{|c|c|c|c|c|c|}
\hline & $\begin{array}{l}\text { Qab Elias/ Houch } \\
\text { El Eiyyedeh }\end{array}$ & 1 & $1.1-2.7$ & 1.9 & \\
\hline $\begin{array}{l}\text { North } \\
\text { Bekaa }\end{array}$ & Machari El Qaa & 3 & $0.0-0.7$ & 0.3 & 66.7 \\
\hline $\begin{array}{l}\text { South } \\
\text { Lebanon }\end{array}$ & Majidiyeh & 1 & $0.1-0.3$ & 0.2 & 100 \\
\hline $\begin{array}{l}\text { Central } \\
\text { Bekaa }\end{array}$ & Marej & 4 & $0.0-2.2$ & 0.7 & \multirow{6}{*}{91.6} \\
\hline \multirow[b]{6}{*}{ Chouf } & Elrawda & 2 & $0.0-2.6$ & 2.1 & \\
\hline & Miksy & 1 & $0.7-0.9$ & 0.8 & \\
\hline & Jdita & 1 & $2.1-3.9$ & 3.0 & \\
\hline & $\begin{array}{l}\text { Zahle-Kaa El } \\
\text { Rim }\end{array}$ & 3 & $1.0-5.1$ & 2.2 & \\
\hline & Zahle El Fayda & 1 & $1.1-1.3$ & 1.2 & \\
\hline & Kfarselwen & 2 & $2.7-5.1$ & 3.8 & \multirow{5}{*}{100} \\
\hline & Jwar El Hawz & 1 & $1.9-3.8$ & 2.9 & \\
\hline & Barouk & 3 & $0.7-3.4$ & 1.7 & \\
\hline & $\begin{array}{l}\text { Barouk El } \\
\text { Ramleh } \\
\end{array}$ & 1 & $1.1-1.4$ & 1.3 & \\
\hline & $\begin{array}{l}\text { Barouk El } \\
\text { Ghafar }\end{array}$ & 1 & $0.4-0.5$ & 0.5 & \\
\hline \multirow[t]{2}{*}{ Akkar } & Kabiit & 4 & $0.3-2.3$ & 1.5 & \multirow{2}{*}{100} \\
\hline & Ammouaa & 6 & $1.2-2.9$ & 1.9 & \\
\hline \multirow[t]{2}{*}{ Jbeil } & Afqa & 1 & 0.0 & 0.0 & \multirow{2}{*}{80} \\
\hline & Tannourine & 4 & $1.0-2.8$ & 1.6 & \\
\hline Bcharreh & Bcharreh & 9 & $0.6-4.6$ & 2.4 & 100 \\
\hline Total & 31 & 53 & $0.0-16.0$ & 1.1 & 88.7 \\
\hline
\end{tabular}

\section{Soil survey in peach orchards}

The occurrence and range of nematodes in peach orchards are presented in Table 4. Forty two soil samples were collected from around peach fruit trees in different parts of Lebanon. Almost all of the collected soil samples (97.6\%) were infested with nematodes. The level of infestation of soil sample was ranging between 0.1 and 27.9 nematodes per $\mathrm{g}$ soil (Table 4). Soil samples collected from Maten, Kesrwan, west Bekaa, north Bekaa, south Lebanon, Akkar, Tannourine and Chouf areas were all infested with nematodes (100\%). The level of infestation with nematodes population varied from one area to another. The highest level was observed (18.4-27.9) in Qoub Elies-Talleh, west Bekaa. While the lowest infestation (0.0-0.1) was detected in a soil sample collected from Taalabeya, Central Bekaa. No nematodes infection was detected in Taalabeya area. Microscopic identification revealed several genera were present in the soil samples including: Meloidogyne spp., Tylenchulus, Xiphinema, Rotylenchus and Pratylenchus. Xiphinema sp. was the most occurring species in the collected soil samples. 


\section{Soil survey in nectarine orchards}

Twenty one soil samples were collected from nectarine orchards. The results revealed that all the collected soil samples were infected with nematodes. The level of infestation of soil samples ranged between 0.4 and 20.1 nematodes per $g$ soil (Table 5). The level of infestation with nematodes population varied from one area to another. The highest level was observed (15.1-20.1) in Qoub Elies-Talleh, west Bekaa, whereas the lowest infestation (0.4-5.2) was detected in soil samples collected from El-Qaa, north Bekaa. Microscopic identification revealed that several genera were also present in the soil samples including: Meloidogyne spp., Tylenchulus, Xiphinema, Rotylenchus, Longidorus and Pratylenchus. Pratylenchus spp. had the highest incidence among other genera.

TABLE 4

Numbers of Plant-Parasitic Nematodes in Soil Samples Collected from Different Locations in Peach Orchards

\begin{tabular}{|c|c|c|c|c|c|}
\hline Region & Area & $\begin{array}{c}\text { No of } \\
\text { samples }\end{array}$ & $\begin{array}{l}\text { Range of } \\
\text { nematodes } \\
\text { in } 1 \text { g/soil }\end{array}$ & Mean & $\begin{array}{c}\text { Infection } \\
\%\end{array}$ \\
\hline \multirow{2}{*}{ Maten } & Wede El Karem & 1 & $1.7-2.0$ & 1.8 & \multirow{2}{*}{100} \\
\hline & Ain El Qabou & 1 & $0.2-0.2$ & 0.2 & \\
\hline \multirow{4}{*}{ Keserwen } & Qlayaat & 1 & $0.3-0.7$ & 0.5 & \multirow{4}{*}{100} \\
\hline & Blat Kfardebyen & 1 & $0.8-1.4$ & 1.0 & \\
\hline & Choum Kfardebyen & 1 & $1.0-1.4$ & 1.2 & \\
\hline & Mrah Cheded Kfardebyen & 1 & $0.5-2.0$ & 1.2 & \\
\hline \multirow{6}{*}{ West Bekaa } & Qab Elias / Hawch EI & 3 & $0.6-10.7$ & 5.1 & \multirow{6}{*}{100} \\
\hline & Qab Elias / El Talleh & 1 & $8.6-10.6$ & 9.6 & \\
\hline & Qab Elias /Talleh & 1 & $18.4-27.9$ & 23.1 & \\
\hline & Qab Elias /EI Chaabeh & 1 & $11.8-14.9$ & 13.3 & \\
\hline & Qab Elias / El Bayda & 3 & $3.8-8.4$ & 5.2 & \\
\hline & Qab Elias / El Jabal & 1 & $2.2-3.0$ & 2.6 & \\
\hline $\begin{array}{l}\text { North } \\
\text { Bekaa }\end{array}$ & Qaa & 6 & $0.8-5.7$ & 2.4 & 100 \\
\hline South & Ain Arab & 1 & $5.0-5.3$ & 5.1 & 100 \\
\hline \multirow{3}{*}{$\begin{array}{l}\text { Central } \\
\text { Bekaa }\end{array}$} & Taalabeya & 5 & $0.0-1.0$ & 0.3 & \multirow{3}{*}{90} \\
\hline & Jdita & 3 & $1.6-7.0$ & 3.8 & \\
\hline & Saadneyil & 2 & $0.8-12.3$ & 9.9 & \\
\hline \multirow{2}{*}{ Chouf } & Kfarselwen & 1 & $8.9-15.9$ & 12.4 & \multirow{2}{*}{100} \\
\hline & Barouk & 5 & $0.6-3.7$ & 2.0 & \\
\hline \multirow{2}{*}{ Akkar } & Hrar & 1 & $3.5-3.6$ & 3.6 & \multirow{2}{*}{100} \\
\hline & Fnaydek & 1 & $1.8-2.5$ & 2.1 & \\
\hline Tannourine & Tanourine Harisa & 1 & $2.5-4.0$ & 3.3 & 100 \\
\hline Total & 22 & 42 & $0.0-15.9$ & 2.61 & 97.6 \\
\hline
\end{tabular}




\section{Soil survey in plum orchards}

Only 6 soil samples were collected in the survey on plum trees in different parts.

All the collecteded soil samples were infested with nematodes. The level of infestation of soil samples ranged between 1.4 and 5.8 nematodes per g soil (Table 6). Soil samples collected from west Bekaa, central Beqaa and South Lebanon regions were all infested with nematodes $(100 \%)$. The level of infestation with nematodes population was varying from one area to another. The highest level was observed (5.7-5.9) in Qab Elias / El Hamra, west Bekaa. While the lowest infestation (1.4-3.4) was detected in soil sample collected from Qaa, north Beqaa. Microscopic identification revealed that several genera were present in the soil samples including: Meloidogyne spp., Longidorus, Tylenchulus, Xiphinema, and Rotylenchus. Other unidentified species were also present in the same samples.

TABLE 5

Number of Plant-Parasitic Nematodes in Soil Samples Collected from Different Locations in Nectarine Orchards

\begin{tabular}{|c|c|c|c|c|c|}
\hline Region & Area & $\begin{array}{c}\text { No of } \\
\text { samples }\end{array}$ & $\begin{array}{c}\text { Range of } \\
\text { nematodes in } \\
1 \mathrm{~g} / \mathrm{soil}\end{array}$ & Mean & $\begin{array}{c}\text { Infection } \\
\%\end{array}$ \\
\hline \multirow{4}{*}{$\begin{array}{l}\text { West } \\
\text { Bekaa }\end{array}$} & Saghbine & 1 & $0.7-8.0$ & 4.3 & \multirow{4}{*}{100} \\
\hline & Qab Elias / EI & 1 & $1.6-4.7$ & 3.2 & \\
\hline & Qab Elias / Talleh & 1 & $15.1-20.1$ & 17.6 & \\
\hline & Qab Elias /EI Jabal & 1 & $3.2-5.3$ & 4.3 & \\
\hline $\begin{array}{l}\text { North } \\
\text { Bekaa }\end{array}$ & El-Qaa & 6 & $0.4-15.6$ & 0.8 & 100 \\
\hline \multirow{2}{*}{$\begin{array}{l}\text { Central } \\
\text { Bekaa } \\
\end{array}$} & El Marej & 4 & $0.6-5.2$ & 1.9 & \multirow{2}{*}{100} \\
\hline & Kfarzabad & 6 & $0.75-9.8$ & 4.4 & \\
\hline $\begin{array}{l}\text { South } \\
\text { Lebanon }\end{array}$ & Majidiyeh & 1 & $1.5-2.1$ & 1.8 & 100 \\
\hline Total & 8 & 21 & $0.4-20.1$ & 1.8 & 100 \\
\hline
\end{tabular}

TABLE 6

Numbers of Plant-Parasitic Nematodes in Soil Samples Collected from Different Locations in Plum Orchards

\begin{tabular}{|c|c|c|c|c|c|}
\hline Area & Region & $\begin{array}{c}\text { No of } \\
\text { samples }\end{array}$ & $\begin{array}{c}\text { Range of } \\
\text { nematodes } \\
\text { in } 1 \text { g/soil }\end{array}$ & Mean & $\begin{array}{c}\text { Infection } \\
\%\end{array}$ \\
\hline Qaa & North Bekaa & 2 & $1.4-3.4$ & 2.4 & 100 \\
\hline Majidiyeh & South & 1 & $2.1-2.2$ & 2.2 & 100 \\
\hline Meksy/ Jdita & \multirow{2}{*}{$\begin{array}{l}\text { Central } \\
\text { Beqaa } \\
\end{array}$} & 1 & $3.1-4.2$ & 3.7 & 100 \\
\hline Zahle / EI Fayda & & 1 & $2.7-4.8$ & 3.8 & 100 \\
\hline Qab Elias / El & West Beqaa & 1 & $5.7-5.9$ & 5.8 & 100 \\
\hline Total & 4 & 6 & $2.1-4.8$ & 3.0 & 100 \\
\hline
\end{tabular}


Soil survey in almond and apricot orchards

In the survey of apricot and almond fruit tree orchards 15 soil samples were collected. The level of infestation ranged between 0.7 and 5.6 nematodes per g soil (Table 7). Soil samples Keserwen, Akkar and South Lebanon areas were all infested with nematodes $(100 \%)$. The level of infestation with nematodes population varied from one area to another. The highest level was observed (6.0-6.4) in Hrar, Akkar, whereas the lowest infestation (0.40.7 ) was detected in soil samples collected from Fnaydek, Akkar. No nematodes infection was obtained in some samples collected from Fnaydek area from both almond and apricot orchards. Microscopic identification revealed several genera were present in the soil samples including: free living nematodes, plant parasitic nematodes; root-knot nematodes (Meloidogyne spp.), Tylenchulus, Xiphinema, Rotylenchus and Pratylenchus spp.

TABLE 7

Numbers of Plant-Parasitic Nematodes in Soil Samples Collected from Different Locations in Almond and Apricot Orchards

\begin{tabular}{|l|l|l|c|c|c|c|}
\hline Region & Area & Type & $\begin{array}{c}\text { No of } \\
\text { samples }\end{array}$ & $\begin{array}{c}\text { Range of } \\
\text { nematodes } \\
\text { in 1 g soil }\end{array}$ & Mean & $\begin{array}{c}\text { Infection } \\
\%\end{array}$ \\
\hline Keserwen & Zaatreh & almond & 2 & $0.7-3.8$ & 2.9 & 100 \\
\hline \multirow{3}{*}{ Akkar } & Fnaydek & apricot & 4 & $0.0-0.7$ & 0.7 & \multirow{2}{*}{50} \\
\cline { 2 - 6 } & Hrar & almond & 3 & $6.0-6.4$ & 6.2 & \\
\cline { 2 - 6 } & Fnaydek & almond & 3 & $0.0-0.4$ & 0.4 & \\
\hline South & Majidiyeh & apricot & 4 & $3.4-5.6$ & 4.5 & 100 \\
\hline Total & $\mathbf{5}$ & & 15 & $0.0-6.4$ & 1.0 & 66.6 \\
\hline
\end{tabular}

TABLE 8

Numbers of Plant-Parasitic Nematodes in Soil Samples Collected from Various Locations in Pear and Quince Orchards*

\begin{tabular}{|l|l|c|c|c|c|}
\hline \multicolumn{1}{|c|}{ Region } & \multicolumn{1}{|c|}{ Area } & $\begin{array}{c}\text { Number of } \\
\text { samples }\end{array}$ & $\begin{array}{c}\text { Range of } \\
\text { nematodes in 1 } \\
\text { g of soil }\end{array}$ & Mean & $\begin{array}{c}\text { Infection } \\
(\boldsymbol{\%})\end{array}$ \\
\hline Maten & Sannine & 1 & 0.0 & 0.0 & 0.0 \\
\hline South Lebanon & Majidiyeh & 1 & $0.6-1.0$ & 0.8 & 100 \\
\hline Chouf & Kfarselwen & 1 & $4.3-4.8$ & 4.6 & 100 \\
\hline Bcharreh & Bcharreh & 1 & $0.2-0.2$ & 0.2 & 100 \\
\hline *West Bekaa & Qoub Elias & 2 & $2.7-9.4$ & 6.1 & 100 \\
\hline *South Lebanon & Majidiyeh & 1 & $0-0.3$ & 0.2 & 100 \\
\hline Total & \multicolumn{6}{|c}{} & 7 & $0.0-9.4$ & 2.0 & 85.7 \\
\hline
\end{tabular}




\section{Soil survey in pear and quince orchards}

Seven soil samples were collected from different areas, four from pear orchards and two from quince orchards. Five out of seven were infested with nematodes. The average number of nematodes varied from one area to anther. The highest number ( 9.4 nematodes in 1 $\mathrm{g}$ soil) was detected in Qab Elias area in quince orchard and 4.8 nematodes in $1 \mathrm{~g}$ soil in pear orchard in Kfarselwen (Table 8).

The impact of different plant materials on nematodes mortality are presented in Table 9. All the treatments used had significant effect $(P<0.05)$ on nematode development in comparison to the control. Not all the treatments showed similar effect against nematodes. The results revealed that carbofuran (nematicide) was the most effective control measure $(88.48 \%)$ in comparison with plant materials. Allium sativum gave the highest control (76.52\%) among the tested plant materials followed by Tageta patula (72.0\%), Cucurbit maxima $(71.84 \%)$ and Inula viscosa $(63.96 \%)$. Whereas Origanum syriacum $(55.04 \%)$ and Thymus capitatus $(53.72 \%)$ were less effective in comparison to the rest of tested plant materials.

TABLE 9

The Effect of Chopped Plant Materials on Plant Parasitic Nematodes Mortality in Pot Experiment

\begin{tabular}{|l|c|c|c|c|c|c|c|c|}
\hline \multirow{2}{*}{ Treatments } & \multicolumn{7}{|c|}{ Number of nematodes $\mathbf{1}$ g soil } \\
\cline { 2 - 9 } & $\mathbf{0}$ day & $\mathbf{2 0}$ day & $\mathbf{4 0}$ day & $\mathbf{6 0}$ day & $\mathbf{8 0}$ day & $\mathbf{1 0 0}$ day & Mean & $\begin{array}{c}\text { Control } \\
\text { \% }\end{array}$ \\
\hline $\begin{array}{l}\text { Allium } \\
\text { sativum }\end{array}$ & $25^{\mathrm{a}}$ & $16.2^{\mathrm{b}}$ & $1.5^{\mathrm{d}}$ & $3.76^{\mathrm{b}}$ & $3.04^{\mathrm{d}}$ & $4.82^{\mathrm{d}}$ & 5.87 & 76.52 \\
\hline $\begin{array}{l}\text { Tageta } \\
\text { patula }\end{array}$ & $25^{\mathrm{a}}$ & $12.06^{\mathrm{b}}$ & $7.24^{\mathrm{c}}$ & $8.18^{\mathrm{b}}$ & $4.56^{\mathrm{d}}$ & $2.97^{\mathrm{d}}$ & 7.0 & 72.0 \\
\hline $\begin{array}{l}\text { Cucurbit } \\
\text { maxima }\end{array}$ & $25^{\mathrm{a}}$ & $6.09^{\mathrm{c}}$ & $9.38^{\mathrm{b}}$ & $13.22^{\mathrm{b}}$ & $1.56^{\mathrm{d}}$ & $4.99^{\mathrm{d}}$ & 7.04 & 71.84 \\
\hline $\begin{array}{l}\text { Inula } \\
\text { viscosa }\end{array}$ & $25^{\mathrm{a}}$ & $13.46^{\mathrm{b}}$ & $8.26^{\mathrm{b}}$ & $7.51^{\mathrm{b}}$ & $6.10^{\mathrm{d}}$ & $9.74^{\mathrm{b}}$ & 9.01 & 63.96 \\
\hline $\begin{array}{l}\text { Origanum } \\
\text { syriacum }\end{array}$ & $25^{\mathrm{a}}$ & $19.85^{\mathrm{a}}$ & $10.49^{\mathrm{b}}$ & $9.79^{\mathrm{b}}$ & $10.32^{\mathrm{b}}$ & $5.77^{\mathrm{d}}$ & 11.24 & 55.04 \\
\hline Thymus & $25^{\mathrm{a}}$ & $12.70^{\mathrm{b}}$ & $28.47^{\mathrm{a}}$ & $9.0^{\mathrm{b}}$ & $4.33^{\mathrm{d}}$ & $3.34^{\mathrm{d}}$ & 11.57 & 53.72 \\
\hline Carbofuran & $25^{\mathrm{a}}$ & $5.02^{\mathrm{d}}$ & $3.78^{\mathrm{d}}$ & $1.85^{\mathrm{d}}$ & $2.68^{\mathrm{d}}$ & $1.05^{\mathrm{d}}$ & 2.88 & 88.48 \\
\hline Control & $25^{\mathrm{a}}$ & $27.60^{\mathrm{a}}$ & $30.02^{\mathrm{a}}$ & $31.24^{\mathrm{a}}$ & $33.1^{\mathrm{a}}$ & $34.5^{\mathrm{a}}$ & 31.33 & 125.32 \\
\hline
\end{tabular}

The data represent the mean of two experiments (Tukey test). ${ }^{\mathrm{a}, \mathrm{b}, \mathrm{c}, \mathrm{d}}$ Data followed by the same letter in the same column are not significantly different at $P<0.05$. 


\section{DISCUSSION}

Nematodes are microscopic worm-like organisms that attack plant roots. Damaged roots result in a reduced uptake of nutrients and water. Field surveys revealed for the first time the wide-spread distribution of different nematodes throughout the surveyed area of Lebanon. A total 308 soil samples were collected from 10 different crops. Almost all surveyed areas showed infection with nematodes. Eighteen out of 308 soil samples were free of nematodes $(5.8 \%)$. The level of soil infestation varied from one area to another. The number of nematodes ranged between 0.1 and 28 per $1 \mathrm{~g}$ soil in cherry orchards, and from 0.1 to 16.0 nematodes per $1 \mathrm{~g}$ soil in apple orchards. It was estimated that $35 \%$ of California's almond and fresh stone fruit acreage is infested with plant parasitic nematodes, such as: ring, lesion, and on some rootstocks, root knot nematodes (McKenry, 1988). In Lebanon, the economic effect of plant parasitic nematodes on stone fruits has not been established. The current survey also discovered the presence of several economically important plant parasitic nematodes on cherry and apple and other crops including root-knot nematodes (Meloidogyne spp.), Tylenchulus, Xiphinema, Rotylenchus, Pratylenchus, Radopholus and Longidorus. The ring nematode has been especially important in sandy soil textures where it induces infection with the bacterial canker complex in trees less than 9 years old (Nyczepir, 1991). In this survey, no ring nematodes were observed using microscopic identification. A recent survey in banana plantations also detected several species of plant parasitic nematodes (Ibrahim et al., 2012). High densities of ring nematodes Criconemella xenoplax and incidences of bacterial canker of peach (Prunus persica) and almond (Prunus dulcis) tend to occur in sandy soils (Brittain \& Miller, 1976; Ogawa \& English, 1991). Paratylenchus spp. are generally associated with various fruit species and may be responsible for damage to their root system (Nyczepir \& Halbrendt, 1993). Xiphinema, and Longidorus spp. are phytopathologically important as a vector of Arabis mosaic virus (Harrison \& Cadman, 1959; Jha \& Posnette, 1959; 1961) and Strawberry latent ringspot virus (Lister, 1964), Grapevine fanleaf virus (Cohn et al., 1970), with an ability to transmit these viruses to fruit trees (Anonymous, 2001a; 2001b). Xiphinema and Longidorus species were observed in several soil samples which could cause several problems on a wide range of crops. In spite of the lack of information on the phytopathological importance of the other species, all these nematodes may be considered as components of pathological and stress factors on trees (Lišková et al., 2007). The size of samples vary from region to region because most of stone plantations are not located in one area, therefore it's difficult for collecting enough samples. Concerning the infected areas, however, Kfarselwen showed the highest infection (mean 6.0) on cherry, and in Qaroun on apple (mean 9.0). Whereas Bcharre had the lowest infection (mean 0.1) on cherry and in Barouk El Ghafar (mean 0.5) on apple. The low infestation in some area with nematodes could be due to the advanced agricultural practices or to the intensive use of pesticides. In many cases, plant parasitic nematodes populations occur in low numbers to cause serious plant injury especially with mature orchards. Serious damage can be visible on root stock or very young trees. Nematodes from soil samples or infected plant parts must be extracted, identified, and counted in order to determine if and which nematode species are causing poor growth. Knowing the number of plant parasitic nematodes present in soil helps to determine control strategies. If the population level is high enough to cause economic damage at or above the "economic threshold density" for that species, then application of control strategies is recommended. It is advisable to promote root growth with the use of a root stimulant. Mulch on top of the soil or the addition of any organic material will be of great benefit. None of these additions will control high nematode numbers, however, in the long term the number of nematodes will decline thereby reducing damage to the crop and encouraging root growth 
and natural enemies (beneficial soil organisms). The mode of action of plant extracts on nematodes is not fully understood. Nematodes are attracted to marigold roots but when they invade them, the root releases ozone to kill them (Ogden, 1997). Moreover, French marigold (Tagetes patula) was also shown to be the most effective type in lowering root-knot nematode populations (Ogden, 1997). Belcher \& Hussey (1977) found that T. patula acted as a trap crop to $M$. incognita, but prevented giant cell initiation. On the other hand, a minimum concentration of $1 \mathrm{mg}$ liter $^{-1}$ of $A$. sativum $\mathrm{L}$. and $F$. vulgare $\mathrm{L}$. significantly decreased the emergence of juveniles of $M$. incognita to $7.6 \%$ and $25 \%$ respectively (Ibrahim \& Traboulsi, 2009). In our study marigold extract showed significant reduction (72\%) in nematodes population. Leaf powder of rock fleabane (Inula viscose) at a concentration of $0.1 \%$ in sand reduced hatching of second-stage juveniles of $M$. javanica and the citrus nematode (Tylenchulus semipenetrans) with the stem-bulb nematodes (Ditylenchus dipsaci) being unaffected (Oka et al., 2001). Similar affect was obtained in our investigation using the same plant extracts. Clove extract and Nimbecidine did not show any potential for the control of Aphelenchoides fragariae on its host (Jagdale \& Grewal, 2002). Gupta and Sharma (1991) indicated that garlic extract had increased larval mortality by $88.64-98.88 \%$ at $0.05-10 \%$ concentration and it increased with increased exposure time. Also, Nath et al. (1982) found that garlic extract had 100\% lethal effect on $\mathrm{J} 2$ of $M$. javanica and $100 \%$ reduction of root penetration, while Shady and Soliman (2008) reported that the application of chopped garlic cloves $(80 \mathrm{mg} /$ tree) gave the highest reduction rate (85\% compared to the control treatment) in nematode population of $M$. incognita in soil and roots of grapevine tree. According to Stoll and Seebeck (1950) the nematicidal activity of garlic is attributed to allicin, diallyl disulfide, ammonia and pyruvic acid. However, Chitwood (2002) proved that allicin at a concentration of $25 \mu \mathrm{g} / \mathrm{ml}$ inhibited penetration of roots by $M$. incognita juveniles by $50 \%$ and was not phytotoxic. In the current study A. sativum gave significant control (76.52\%) in pot experiment. Also, we can suppose that the six dried plant materials of C. maxima, $T$. capitatus, T. patula, O. syriacum, I. viscosa, and A. sativum have nematicidal effect in controlling ectoparasitic nematodes with different ratios and different mechanisms. Further investigations are required, that could lead to the exploitation of the natural biocidal activity of plant extracts against nematodes as an environmentally benign control measure. In conclusion, the results of this investigation are very important to determine the distribution of plant parasitic nematodes in fruit orchards in Lebanon, in particular species that are virus vectors. This knowledge is essential for developing nematodes management and quarantine measurers.

\section{ACKNOWLEDGEMENTS}

The authors acknowledge the CNRS Lebanon for financial support.

\section{REFERENCES}

Anonymous 2001a. Certification scheme for cherry. OEPP/EPPO Bulletin, 31: 447-461.

Anonymous $2001 \mathrm{~b}$. Certification scheme for almond, apricot peach and plum. OEPP/EPPO Bulletin, 31: 463-478.

Belcher, J.V., Hussey, R.S. 1977. Influence of Tagets patula and Archis hypogeaon Meloidogyne incognita. Plant Disease Reporter, 61: 525-528.

Brittain, J.A., Miller, R.W. 1976. Managing peach tree short life in South Carolina. Circular 568, Clemson, SC: Clemson University Extension. 
Chitwood, D.J. 2002. Phytochemical based strategies for nematode control. Nematology Laboratory. Annual Review Phytopathology, 40: 221-249.

Cohn, E., Tanne, E., Nitzany, F.E. 1970. Xiphinema italiae, a new vector of grapevine fanleaf virus. Phytopathology, 60: 181-182.

Duncan, L.W. 2005. Nematodes parasites of citrus. In: Plant parasitic nematodes in subtropical and tropical agriculture, pp. 437-466.

Fenwick, D.W. 1949. Investigations on the emergence of larvae from cysts of the potato-root eelworm, Heterodera rostochiensis. I. Technique and variability. Journal of Helminthology, 23: 157-170.

Gupta, R., Sharma, N.K. 1991. Nematicidal properties of garlic (Allium sativum). Indian Journal of Nematology, 21: 14-18.

Harrison, B.D., Cadman, C.H. 1959. Role of a dagger nematode (Xiphinema sp.) in outbreaks of plant diseases caused by arabis mosaic virus. Nature (London), 184: 1624-1626.

Hooper, D.J, Ibrahim S.K. 1994. Aphelenchoides nechaleos n.sp. and A. paranechaleos n.sp. (Nematoda: Aphelenchoididae) from rice plants. Fundam. Appl. Nematol., 17: 153-160.

Ibrahim, S.K., Hooper, D.J. 1994. Aphelenchoides varicaudatus n.sp. (Nematoda: Aphelenchoididae). Afro-Asian J. of Nematology, 4: 210-214.

Ibrahim, S.K., Traboulsi, A.F., El-Haj, S. 2006. Effects of essential oils and plant bioproduts against Meloidogyne incognita. Phytopathol. Mediterr., 45: 238-246.

Ibrahim, S.K., Traboulsi, A.F. 2009. The impact of solarisation integrated with plant biofermentation on root knot nematodes. Lebanese Science Journal, 10(2): 59-69.

Ibrahim, S.K., Ibrahim, L., Ismail, A., Kayal, M., Ghanem, H., Rammel, S. 2011. Differentiation of different species of Origanum and Thymus using proteins and isoenzymes profile. International Journal of Botany, 7: 283-288.

Ibrahim, S.K., Ibrahim, L., Choueiry, I. 2012. The occurrence of burrowing nematodes on banana in Lebanon and their control using plant extracts and essential oil of Origanum sp. World Research Journal of Entomology and Nematology, 1: 01-06.

Jagdale, G.B., Grewal, P.S. 2002. Identification of alternatives for the management of foliar nematodes in floriculture. Pest Management Science, 58: 451-458.

Jha, A., Posnette, A.F. 1959. Transmission of a virus to strawberry plants by a nematode (Xiphinema sp.). Nature (London), 184: 962-963.

Jha, A., Posnette, A.F. 1961. Transmission of arabis mosaic virus by Xiphinema diversicaudatum (Micol.). Virology, 13: 119-123.

Knight, K.W.L. 2001. Plant parasitic nematodes associated with six subtropical crops in New Zealand. New Zealand Journal of Crop and Horticultural Science, 29: 267-275.

Lišková, M., Sasanelli, N., D’Addabbo, T. 2007. Some notes on the occurrence of plant parasitic nematodes on fruit trees in Slovakia. Plant Protect. Sci., 43: 26-32.

Lister, R.M. 1964. Strawberry latent ringspot: a nematode-borne virus. Annals of Applied Biology, 54: 167-176.

Marban-Mendoza, N, Jeyaparakash, A, Jansson, H.B., Damon, R.A. Jr. and Zuckerman, B.M. 1987. Control of root-knot nematodes on tomato by lectins. J. Nematol., 19: 331-335.

McKenry, M.V. 1988. Nematodes of stone -fruits, California, P761-77o. In: N.F. Childers and W.B. Sherman (eds). The peach horticultural publication, Gainsville, Florida.

Nath, A., Sharma, N.K., Bhardwaj, S. Thapa, C.D. 1982. Nematicidal properties of garlic. Nematologica, 28: 253 -255.

Nyczepir, A.P. 1991. Nematode management strategies in stone fruits in the United States. Journal of Nematology, 23: 334-341. 
Nyczepir, A.P., Halbrendt, J.M. 1993. Nematode pests of deciduous fruit and nut trees. In: Evans, K., Trudgill, D.L., Webster, J.M. (eds): plant parasitic nematodes in temperate agriculture, CAB International, Wallingford, p. 381-425.

Nyczepir, A.P., Becker, J.O. 1998. Fruit and citrus trees. In: Barker, K.R., Pederson, G.A., Windham, G.L. (eds): plant and nematode interactions. American Society of Agronomy, Madison, pp. 637-684.

Ogawa, J.M., English, H. 1991. Diseases of temperate zone tree fruit and nut crops. Publication 3345, Oakland, CA: University of California, Division of Agriculture and Natural Resources.

Ogden, S. 1997. Marigolds bite back. National Gardening, March-April, 21 p.

Oka, Y., Ben-Daniel, B., Cohen, Y. 2001. Nematicidal activity of powder and extracts of Inula viscosa. Nematology, $3: 735-742$.

Okoko, F.J., Nwafor, O.E., Ejechi, B.O. 1999. Growth inhibition of tomato-rot fungi by phenolic acids and essential oil extracts of pepperfruit (Dennetia tripetala). Food Res. Inter., 3: 395-399.

Saltaren, G., Luis, F., de Varon Agudelo Francia, H., de la Torre Fernando, M. 1999. Nematodes associated with crops of avocado (Persea americana Mill). Fitopatologia Colombiana, 22: 68-73.

Sasser, J.N., Freckman, D.W. 1987. A world prospective on nematology: the role of the society. In: Vistas on Nematology, edited by J.A., Veech and D.W., Dickson. Society of Nematologists, Hyattsville, MD, pp. 7-14.

Shady, A.M., Soliman, M. 2008. Studies on surveying nematodes, host suitability and control of Meloidogyne incognita on grapevine by certain com-pounds with reference to nematode infestation and yield. Plant Pathology Research Institute, A.R.C., Giza, Egypt.

Speijer, P.R., de Waele, D. 1997. Screening of Musa germplasm for resistance and tolerance to nematodes. INIBAP Technical Guidelines 1, INIBAP, Montpellier, France, 42p.

Stoll, A., Seebeck, E. 1950. Specific constituents of garlic. Scientia Pharmaceutica, 18: 6179.

Thackray, D.J, Wratten, S.D., Edwards, P.J., Niemyer, H.M. 1990. Resistance to the aphids Sitobion avenae and Rhopalosiphon padi in Gramineae in relation to hydroxamic acid levels. Annals of Applied Biology, 116: 573-582.

Tudor, M.E., McKenry, M.V. 1989. Unraveling the various nematicidal activities within aqueous extracts of plant refuse. Journal of Nematology, 21(4): 592 (Abst.).

Zehr, E.I., Lewis, S.A. and Bonner, M.J. 1986. Some herbaceous hosts of the ring nematode (Criconemella xenoplax). Plant Disease, 70: 1066-1069. 